\title{
Rescheduling Based Congestion Management Method Using Hybrid Grey Wolf Optimization - Grasshopper Optimization Algorithm in Power System
}

\author{
Rupam Gupta Roy \\ Department of Electronics and Instrumentation Engineering \\ National Institute of Technology, \\ Agartala, Tripura, India \\ royrupamgupta@gmail.com
}

\begin{abstract}
Safe and incessant power flow is considered as a serious problem which has to be corrected in the transmission line. Actually, rescheduling based Congestion Management is deliberated as the capable solutions for the feature. Still, the technique faces problems based on the cost of rescheduling. A huge number of research studies have been identified hitherto to resolve the problems in Congestion Management. In addition, optimization methods play a very important role to solve this problem. In this case, this work develops a novel rescheduling based Congestion Management. technique that integrates a novel method; Grey Wolf Optimization (GWO) and Grasshopper Optimization Algorithm (GOA) (GWO-GOA) which optimizes generating power of augmented generators using the bus system. The proposed GWO-GOA method is the hybridization of two methods such as GWO and GOA, which aspires to manage the congestion through minimized rescheduling cost. Furthermore, the proposed technique evaluates its performance with the other existing techniques based on rescheduling stratagem regarding the analysis of cost it shows the effectiveness of proposed methods over other existing algorithms.
\end{abstract}

Keywords: Congestion Management; Power System; Rescheduling; Bus System; Optimization Algorithm

\section{Nomenclature}

\begin{tabular}{ll}
\hline Abbreviations & Descriptions \\
\hline RED & Relative Electrical Distance \\
CM & Congestion Management \\
ISOs & Independent system operators \\
GSF & Generator Sensitivity Factor \\
MOPSO & Multi-Objective \\
GLCM & Generation and Load shedding Cost Minimization \\
PSO & Particle Swarm Optimization \\
PshdM & Load Shedding Minimization \\
BSF & Bus Sensitivity Factor \\
LSE & Load Served Error \\
SWM & Social Welfare Maximization \\
LSM & Load Served Maximization \\
ALO & Ant Lion Optimiser \\
DRPs & Demand Response Programs \\
RED & Relative Electrical Distance \\
CM & Congestion Management \\
ISOs & Independent system operators \\
GSF & Generator Sensitivity Factor \\
MOPSO & Multi-Objective \\
GLCM & Generation and Load shedding Cost Minimization \\
PSO & Particle Swarm Optimization \\
\hline
\end{tabular}

(C) Resbee Publishers 


\begin{tabular}{ll}
\hline PshdM & Load Shedding Minimization \\
BSF & Bus Sensitivity Factor \\
LSE & Load Served Error \\
SWM & Social Welfare Maximization \\
LSM & Load Served Maximization \\
ALO & Ant Lion Optimiser \\
DRPs & Demand Response Programs \\
\hline
\end{tabular}

\section{Introduction}

It is essential to preserve power system security margin in a consistent limitation for incessant power contribute to consumers exclusive of disturbing system. To reduce the line congestion generators encompass to be rescheduled as thermal limitation of transmission lines are predetermined. In the electricity market, it is complicated to ease congestion because of the arbitrary power transaction variation. In the deregulated market numerous techniques have been used to reduce the congestion. In the hybrid electricity market, the exploitation of Sen Transformer for Congestion Management that is a single-core three phase transformer by means of a Y-connected foremost winding in addition to 9 secondary windings and it presents both impedance, and voltage regulation function for independent control of bidirectional reactive and active power, flows in a transmission system [15].

To transmit the electric power the ability of transmission lines is controlled by numerous transfer limits like voltage limit, stability limit and thermal limit,. The power system is considered congested while as a minimum any one of these parameters attains its utmost limit. ISOs encompass a most important responsibility to organize between the diverse market players and to deal with reliable and secured power flow in the seriously loaded conventional transmission infrastructures. An augment in power insist, unpredicted generation outages, unscheduled power flow in the lines, restriction on the structure of new lines, transmission lines tripping or malfunctions of other equipment are a few possible reasons for congestion. Considering reactive power sustain, rescheduling of generation, re-adjustment of demand, and so forth are some choices that might be used to deal with the congestion problem [16].

A new Congestion Management technique was used by the rescheduling of generation and load shedding by means of the voltage-dependent load modeling. Real power generation rescheduling on basis of RED idea was proposed in [7]. Congestion Management efficiency of the market flow approach was stated in [8]. A clusters-based congestion technique that recognizes the cluster of users consistent with their effect on transmission constraints of attention was presented in [9]. Reactive and real power rescheduling through a zonal Congestion Management approach was explained in [10]. The objective models of minimizing generation costs and load shedding involving maximizing social welfare, reducing load shed, and maximizing the load served were explained. In [11], a competent method for Congestion Management is recommended that aspired at altering the generator's output power plan taking into consideration the pumped-storage hydropower plant unit in the power system. An optimization technique was presented following two generators and bus sensitivity factors. The optimal position of pumped-storage hydropower plant unit is recognized exploiting number of bus sensitivity factor and the count of generators contribution to Congestion Management, whom program was altered because of the sensitivity factor of generator.

This work proposes a novel rescheduling based Congestion Management technique through highly proposed soft computing methods. A novel GWO-GOA method is proposed which optimizes the power generation of connected generators in the bus system. The proposed technique aspires in minimizing the congestion yet in minimized cost of rescheduling. The proposed GWO-GOA performance is evaluated with the existing techniques and shows its advantage regarding the definite analysis of performance. 


\section{Literature Review}

In 2018, Fariborz Zaeim-Kohan et al [1] proposed the MOPSO technique that was used for transmission Congestion Management taking into consideration DRPs and rescheduling of generation. Total operation/ cost of DR, emission and rising the transmission lines loading were the objective models of this problem. Utilizing DRPs augments operator power of choice concerning the participation of diminutive consumers in reducing demand. In 2020, Aishvarya Narain et al [2], worked on the congestion in the system might reason uneconomical operation and/else blackouts, interconnected system outage and a disturbance in systems. In up-and-coming competing electricity markets, Congestion Management plays a significant role in the economical operation, stable and secure operation of the power system. Here, numerous significant works of literature proposed for CONGESTION MANAGEMENT were significantly examined. Diverse optimization methods proposed to ease congestion were explained in detail. In 2016, Sadhan Gope et al [3], proposed an optimization technique for Congestion Management by combining computation of 2 factors like GSF and BSF. Optimal positioning of PSHU was recognized exploiting the BSF's value and amounts of contributor's generators for Congestion Management by rescheduling their outputs was decided to exploit GSF's value. The PSHU effect was examined to control transmission congestion that additionally minimizes the cost of congestion and enhances system security. In 2016, S. Surender Reddy et al [4], proposed a novel Congestion Management model exploiting the rescheduling of generation and load shedding, through realistic voltage-dependent load modeling. Moreover, this work presented numerous objective models like including SWM demand response offers, GL Congestion Management, LSM, PshdM, and LSE minimization. Hence, multi-objective optimization was needed and the objectives can be sensibly integrated based upon the loading circumstance. To resolve the proposed Congestion Management problem Multi-objective Strength Pareto Evolutionary method 2+ was used. In 2016, Sumit Verma and Vivekananda Mukherjee [5], presented a generation rescheduling-based technique for Congestion Management exploiting a new ALO algorithm in the electricity market. The efficiency of the proposed method was examined on enhanced IEEE 30-bus, enhanced IEEE 57-bus, and 118-bus test systems. The security constraints namely line loading and load bus voltage effect were integrated into this work. In 2016, Mohammad Mahmoudian Esfahani and Gholam Reza Yousefi [6], presented an optimal real-time transmission Congestion Management approach in a competitive electricity market based on the real-time measurements in a smart power system. Here, this time interval was proposed and stated as congestion clearing time. Moreover, in the proposed method, the congestion clearance time was partitioned into some following subintervals aspired at considering loads and power system deviations throughout the rescheduling process. The proposed technique might be exploited by system operators to acclimatize the real-time Congestion Management process regarding the power system loads and generations or system configuration variations throughout rescheduling and load shedding procedure.

\section{Problem Description in Congestion Management}

The problem is devised based on the hybrid electricity market, in that both pool market features, and bilateral contracts, are involved to be attainable. Moreover, this Congestion Management problem is corrected by the rescheduling of the generator; hence the congestion acquires reduced with the minimized cost of rescheduling [12]. Hence, eq. (1) explains the expression of this whole problem as a constrained minimization function.

Presume, $\Delta \mathrm{P}_{\mathrm{p}}: 0 \leq \mathrm{p} \leq \mathrm{N}_{\mathrm{g}}(\mathrm{MW})$ as power change is to be rescheduled for the cost of $\operatorname{co}\left(\Delta \mathrm{P}_{\mathrm{p}}\right)$ in $\$$

for $\mathrm{p}^{\text {th }}$ generator unit. As in Eq. (1), the minimization problem is represented which is fed to particular constraints.

$$
\mathrm{GE}^{*}=\underset{\Delta \mathrm{P}_{\mathrm{p}} \forall \mathrm{p}}{\arg \min } \sum_{\mathrm{p}=1}^{\mathrm{Ng}} \mathrm{c}\left(\Delta \mathrm{P}_{\mathrm{p}}\right)
$$




\section{(a) Constraint of Generator Capacity [12]:}

$$
\mathrm{P}_{\mathrm{p}}^{\min } \leq \mathrm{P}_{\mathrm{p}} \leq \mathrm{P}_{\mathrm{p}}^{\max }
$$

whereas the quantity of generation in $\mathrm{MW}$ is shown by $\mathrm{P}_{\mathrm{p}}$, that is decided by Eq. (3) if $\Omega \mathrm{P}_{\mathrm{p}}$ indicated the hitherto quantity of generated power.

$$
\mathrm{P}_{\mathrm{p}}=\Omega \mathrm{P}_{\mathrm{p}}+\Delta \mathrm{P}_{\mathrm{p}}
$$

\section{(b) Constraint of Real Power Balance [12]:}

Eq. (4) shows this constraint, whereas total power demand is shown by $P_{\mathrm{DE}}$ and the loss of transmission is shown as $\mathrm{P}_{\mathrm{LO}}$ which is described in Eq. (5), whereas, the coefficients of loss and it is stated as $\mathrm{L}_{\mathrm{qp}}, \mathrm{L}_{0 \mathrm{p}}$ and $\mathrm{L}_{00}$.

$$
\begin{aligned}
& \sum_{\mathrm{p}=1}^{\mathrm{N}_{\mathrm{g}}} \mathrm{P}_{\mathrm{p}}-\left(\mathrm{P}_{\mathrm{DE}}+\mathrm{P}_{\mathrm{LO}}\right)=0 \\
& \mathrm{P}_{\mathrm{LO}}=\sum_{\mathrm{q}=1}^{\mathrm{N}_{\mathrm{g}}} \sum_{\mathrm{p}=1}^{\mathrm{N}_{\mathrm{g}}}\left(\mathrm{P}_{\mathrm{q}} \mathrm{L}_{\mathrm{qp}} \mathrm{P}_{\mathrm{p}}\right)+\sum_{\mathrm{p}=1}^{\mathrm{N}_{\mathrm{g}}}\left(\mathrm{L}_{0 \mathrm{p}} \mathrm{P}_{\mathrm{p}}\right)+\mathrm{L}_{00}
\end{aligned}
$$

\section{(c) Stability Limits:}

Actually, this is the most significant standard for explaining congestion, also, to assure the congestion alleviation too. They are stated in Eq. (6), (7) and (8), whereas $\partial_{\mathrm{i}}^{\min }, \partial_{\mathrm{i}}^{\max }$ are the angle limits and $\left(V_{i}^{\min }, V_{i}^{\max }\right)$ indicates the voltage limits. From generator to load buses both these indicate voltage stability.

$$
\begin{aligned}
& \mathrm{V}_{\mathrm{i}}^{\min } \leq \mathrm{V}_{\mathrm{i}} \leq \mathrm{V}_{\mathrm{i}}^{\max } \\
& \partial_{\mathrm{i}}^{\min } \leq \partial_{\mathrm{i}} \leq \partial_{\mathrm{i}}^{\max } \\
& \mathrm{P}_{\mathrm{ij}}^{2}+\mathrm{R}_{\mathrm{ij}}^{2} \leq\left(\mathrm{M}_{\mathrm{ij}}^{\max }\right)^{2}
\end{aligned}
$$

The certainty: Eq. (8) states the transmission lines do not overload, whereas $\mathrm{M}_{\mathrm{ij}}^{\max }$ shows the limitation of utmost power flow in MVA.

\section{(d) Ramp up Limit [12]:}

Both down and up rescheduling quantity restricts are referred to as ramp up limits. As equivalent down and up costs of rescheduling are deliberated whereas, ramp up limits as stated in Eq. (9).

$$
\Delta \mathrm{P}_{\mathrm{p}}^{\min } \leq \Delta \mathrm{P}_{\mathrm{p}} \leq \Delta \mathrm{P}_{\mathrm{p}}^{\min }
$$

From eq. (1), the optimal rescheduling scheme GE* is attained, by that the congestion is reduced with minimum cost for rescheduling.

\subsection{Cost of Congestion}

The cost of congestion is alleviated by rescheduling generators' active power output. The reality, this rescheduling is obtained also by minimizing else maximizing active power output. However, this increase and a decrease of active power output subjected to charge which is available as price bids. GENCOs do peak price bids. Therefore, rescheduling cost is stated as the congestion cost and it is stated in Eq. (10).

$$
\mathrm{CO}_{\text {total }}=\sum_{j \in \mathrm{N}_{\mathrm{g}}}\left(\mathrm{CO}_{\mathrm{j}} \Delta \mathrm{P}_{\mathrm{p}}^{+}(\mathrm{j})+\mathrm{B}_{\mathrm{j}} \Delta \mathrm{P}_{\mathrm{p}}^{-}(\mathrm{j})\right) \$ / \mathrm{n}
$$

whereas, $\mathrm{CO}_{\text {total }}$ shows the total cost to adapt active power output $(\$ / n), \mathrm{CO}_{\mathrm{j}}: \mathrm{j}=1,2, \ldots, \mathrm{N}_{\mathrm{g}}$ and $\mathrm{B}_{\mathrm{j}}$ shows the price bids to increase the power and minimizing power in $\$ / M W n$ by $j^{\text {th }}$ GENCO, 
generators' active power increment (MW), is stated as $\Delta \mathrm{P}_{\mathrm{p}}^{+}(\mathrm{j})$ and its decrease (MW) is stated as $\Delta \mathrm{P}_{\mathrm{p}}^{-}(\mathrm{j})$. Furthermore, active power change shows dissimilarity among the submitted price in attendance hour (n) from the previous hour $(\mathrm{n}-1)$ stated in Eq. (11).

$$
\Delta \mathrm{P}_{\mathrm{p}}=\left|\mathrm{P}_{\mathrm{p}}(\mathrm{n}-1)-\mathrm{P}_{\mathrm{p}}(\mathrm{n})\right|
$$

whereas $\Delta \mathrm{P}_{\mathrm{p}}$ shows the modify in Active power. The generated active power at $(\mathrm{n}-1)$ is shown in $P_{p}(n-1)$ and the power generated at the present hour is shown as $P_{p}(n)$.

\section{Optimization-based Congestion Management: Proposed Hybrid Method}

\subsection{Objective Function}

The cost $\operatorname{co}\left(\Delta \mathrm{P}_{\mathrm{p}}\right)$ for $\mathrm{j}^{\text {th }}$ generation unit is the summation of total rescheduling cost and infraction level on the voltage of the equivalent transmission line. As in Eq. (12), total cost function which decides the rescheduling scheme.

$$
\operatorname{co}\left(\Delta \mathrm{P}_{\mathrm{p}}\right)=\mathrm{CO}_{\text {total }}+\mathrm{E}_{\text {profile }} \mathrm{CO}_{\text {profile }}+\mathrm{E}_{\text {constraint } \mathrm{s}} \mathrm{CO}_{\text {constraints }}
$$

whereas, $\mathrm{E}_{\text {constraints }}$ and $\mathrm{E}_{\text {profile }}$ shows the cost of penalty forced on violating the voltage profile and constraints. Moreover, $\mathrm{CO}_{\text {profile }}$ is stated in Eq. (13). The generator's least and the utmost voltage is shown as $\mathrm{V}_{\mathrm{p}}^{\min }$ and $\mathrm{V}_{\mathrm{p}}^{\max }$

$$
\mathrm{CO}_{\text {profile }}=\left|\min \left(\mathrm{V}_{\mathrm{p}}-\mathrm{V}_{\mathrm{p}}^{\min }, 0\right)\right|+\left|\min \left(\mathrm{V}_{\mathrm{p}}^{\max }-\mathrm{V}_{\mathrm{p}}, 0\right)\right|
$$

This work aspires to develop a novel rescheduling based Congestion Management technique which simulated in IEEE 14 bus system. It is aimed to attach 3 generators $\left(\mathrm{GE}_{1}, \mathrm{GE}_{2}, \mathrm{GE}_{3}\right)$ to the conventional generator, whereas the generator power $\mathrm{P}_{\mathrm{p}}$ is augmented with the power of the connected generator and indicates as the generator's active power augmentation $\Delta \mathrm{P}_{\mathrm{p}}^{+}(\mathrm{j})$. The generating power is optimized which ranges in $\left(\mathrm{P}_{\mathrm{p}}^{\min }, \mathrm{P}_{\mathrm{p}}^{\max }\right)$. This work develops a novel hybrid optimization method which hybridizes the idea of GWO and ROA for this selection of optimal.

\subsection{Conventional GWO}

In [13], the GWO is the meta-heuristic approach is enthused by imitating the social wolf's class, to explain the procedure of wolf hunting by mathematical techniques. Here, $\alpha$ indicates optimal wolf to indicate the present hunting outcomes, $\beta$ indicates the second optimal solution, $\gamma$ indicates the third optimal solution, and $\omega$ all other solutions. While hunting starts, $\alpha$ superiors hunting the direction of wolves and every wolf decide social class by hunting quality. The formulation can be stated as

$$
\begin{aligned}
& \vec{Y}(t+1)=\vec{Y}_{p}(t)-\vec{A} \cdot \vec{D} \\
& \vec{D}=\left|\vec{C} \cdot \vec{Y}_{p}(t)-\vec{Y}(t)\right| \\
& \vec{A}=a\left(2 \vec{r}_{1}-1\right) \\
& \vec{C}=2 \vec{r}_{2} \\
& a=2\left(1-\frac{t}{T}\right)
\end{aligned}
$$


Whereas, $\vec{Y}$ indicates the gray wolf location, operator, $t$ indicates iteration, and $\vec{Y}_{\mathrm{p}}$ indicates the prey position, - indicates vector multiplication, and $\vec{r}_{1}$ and $\vec{r}_{2}$ indicates 2 arbitrary numbers among [0; 1]. While hunting initiates, individuals will be updated based on the top 3 individuals in the past, as stated below:

$$
\begin{aligned}
& \overrightarrow{\mathrm{Y}}(\mathrm{t}+1)=\frac{\overrightarrow{\mathrm{Y}}_{1}+\overrightarrow{\mathrm{Y}}_{2}+\overrightarrow{\mathrm{Y}}_{3}}{3} \\
& \overrightarrow{\mathrm{Y}}_{1}=\overrightarrow{\mathrm{Y}}_{\alpha}-\mathrm{A}_{1} \cdot \overrightarrow{\mathrm{D}}_{\alpha} ; \overrightarrow{\mathrm{Y}}_{2}=\overrightarrow{\mathrm{Y}}_{\beta}-\mathrm{A}_{2} \cdot \overrightarrow{\mathrm{D}}_{\beta} ; \overrightarrow{\mathrm{Y}}_{3}=\overrightarrow{\mathrm{Y}}_{\gamma}-\mathrm{A} \cdot \overrightarrow{\mathrm{D}}_{\gamma} ; \\
& \overrightarrow{\mathrm{D}}_{\alpha}=\left|\mathrm{C}_{1} \cdot \overrightarrow{\mathrm{Y}}_{\alpha}-\overrightarrow{\mathrm{Y}}\right| ; \overrightarrow{\mathrm{D}}_{\beta}=\left|\mathrm{C}_{2} \cdot \overrightarrow{\mathrm{Y}}_{\beta}-\overrightarrow{\mathrm{Y}}\right| ; \overrightarrow{\mathrm{D}}_{\gamma}=\left|\mathrm{C}_{3} \cdot \overrightarrow{\mathrm{Y}}_{\gamma}-\overrightarrow{\mathrm{Y}}\right|
\end{aligned}
$$

whereas $\vec{Y}(t+1)$ indicates the location after the individuals are updated for iteration $t+1$. At the time of the attack stage and prey search, the consequence of distance is described using Eq. (15) which is arbitrarily highlighted or undermined with diverse parameters A and C, correspondingly.

\subsection{Conventional GOA}

The GOA [14] the updates of every individual are based on 3 factors such as gravity force, social interaction, and wind advection, indicated by S,G and A, correspondingly. Similar to gravity force and wind advection, social interaction is the principal factor that reasons the diverse location of every individual that is described as below:

$$
\begin{aligned}
& S_{i}=\sum_{j=1}^{N} s\left(d_{i j}\right) \hat{d}_{i j} \\
& s(r)=f e^{\frac{-r}{1}}-e^{-r} \\
& \hat{d}_{i j}=\frac{Y_{j}-Y_{i}}{d_{i j}} \\
& r=d_{i j}=\left|Y_{j}-Y_{i}\right|
\end{aligned}
$$

whereas, $\hat{\mathrm{d}}_{\mathrm{ij}}$ indicates a unit vector from $\mathrm{i}^{\text {th }}$ individual to $\mathrm{j}^{\text {th }}$ individual $\mathrm{N}$ indicates the number of individuals, and $\mathrm{d}_{\mathrm{ij}}$ indicates the Euclidean distance among the $\mathrm{i}^{\text {th }}$ individual and the $\mathrm{j}^{\text {th }}$ individual. $\mathrm{f}$ and 1 indicates the parameters exploited to regulate social interaction. It is value state that the distance among 2 individuals should be mapped in the range [1;4]. The wind advection, and gravity force, can be calculated as below:

$$
\begin{aligned}
& N \\
& A_{i}=u \widehat{e}_{w}
\end{aligned}
$$

whereas $\hat{\mathrm{e}}_{\mathrm{w}}$ indicates the unit of the present wind direction and $\hat{\mathrm{e}}_{\mathrm{g}}$ indicates a unit vector towards Earth center, $\mathrm{v}$ indicates a fixed float coefficient, and $\mathrm{g}$ indicates gravitational constant. The aforesaid formulations can be reviewed as an update formulation as below:

$$
Y_{i}=\sum_{j=1}^{N} s\left(d_{i j}\right) \hat{d}_{i j}-g \hat{e}_{g}+u \hat{e}_{w}
$$


To solve a function optimization problem, Eq. (28) have misplaced the capability to discover and expand the search space; therefore, an enhanced update formulation was subsequently presented in [14] that is described as below:

$$
\begin{aligned}
& Y_{i}^{d}=c\left(\sum_{j=1}^{N} c \frac{u_{d}-1_{d}}{2} s\left(d_{i j}\right) \hat{d}_{i j}\right)+\hat{T}_{d} \\
& c=c_{\max }-t\left(\frac{c_{\text {max }}-c_{\text {min }}}{T}\right)
\end{aligned}
$$

whereas $Y_{i}^{d}$ and $\hat{T}_{d}$ indicate, correspondingly, the location of $\mathrm{d}^{\text {th }}$ dimension of $i^{\text {th }}$ individual and optimal up to now individual. $1_{d}$ and $u_{d}$ the lower and upper bounds of $\mathrm{d}$ dimension, correspondingly, $\mathrm{T}$ indicates the total number of iterations and ${ }^{t}$ indicates the current iteration.

\subsection{Proposed Hybrid Technique}

The essential idea of this hybrid approach is to employ the capability of GOA to get away local optima and to employ the capability of GWO to speed up convergence speed of search method to enhance the capability of exploitation and exploration simultaneously. In GWO, $\alpha$ will influence the search trends of $\beta$, and $\gamma$ to enhance the outcomes of majority wolves, at convergence procedure with the intention which can discover a better solution rapidly. Nevertheless, this approach encompasses a similar predicament as the majority of meta-heuristic methods since it might fall into local optima rapidly. In the proposed method, the GOA method is exploited to appraise communication among sets of solutions. While complete solution set moves toward the best solution, pull among solutions in solution sets can get better ability to avoid or jump off local optima and intensification the solution swap is the social association method.

The proposed method will initial initialize the necessary parameters and arbitrarily choose 3 candidates $\overrightarrow{\mathrm{Y}}_{\alpha}, \overrightarrow{\mathrm{Y}}_{\beta}$, and $\overrightarrow{\mathrm{Y}}_{\gamma}$. Subsequently, they will be incessantly updated by Evaluation (), Update (), and Ranking () functions in major loop, and Update () function is a central part of the method that comprises proposed methods. Previous to turning to the proposed technique, to begin the encoding technique is needed. After that, the explanations of the proposed method are defined as follows:

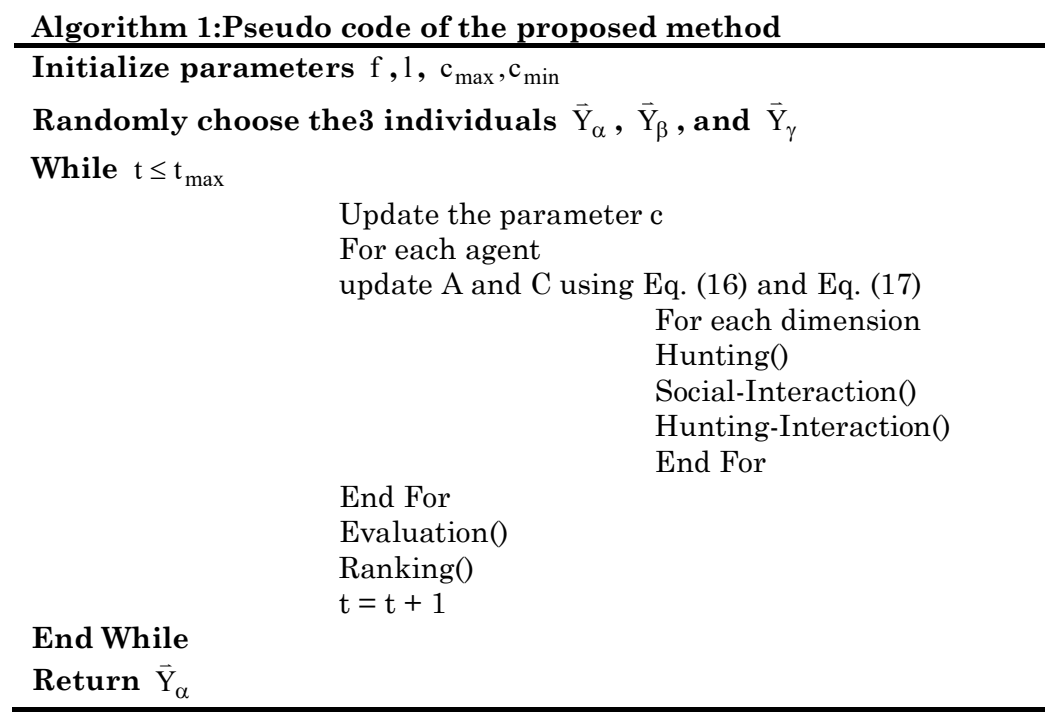


Rescheduling Based Congestion Management Method Using Hybrid Grey Wolf Optimization - Grasshopper Optimization Algorithm in Power System

\section{Results and Discussions}

\subsection{Experimental Set-Up}

The proposed technique has experimented. The simulation was performed in two test bus systems such as IEEE 14 and 30 bus systems. The system consists of loads, transmission lines, capacitor banks, and generators. 3 GENCOs were connected in the IEEE 14 bus system, were connected in the IEEE 30 bus system. By considering this, Tables 1 and 2 exhibit performance analysis of the proposed method. Furthermore, the performance of the proposed rescheduling scheme was evaluated with the conventional techniques such as Particle Swarm Optimization (PSO), Firefly (FF), Grey Wolf Optimization (GWO), and Artificial Bee Colony (ABC) and Whale Optimization Algorithm (WOA) regarding the cost analysis.

\subsection{Performance Analysis}

In this section, the computation time and overall analysis of the proposed method and other rescheduling scheme techniques (both IEEE 14 and 30 bus systems), is shown in Table 1 and 2 .

The overall cost analysis of the proposed method and existing rescheduling based Congestion Management models are summarized in Table 1. Here, three costs such as congestion cost, compensation cost, and the final cost are verified and summarized. Here, the values show that the proposed method obtains minimum cost while evaluated with the existing techniques in the IEEE 14 bus and 30 systems.

In Table 2 the proposed technique obtains minimum computational time, where the residual existing techniques have acquired high computation time is analyzed.

Table 1: Overall Cost Analysis Of Proposed And Existing Techniques

\begin{tabular}{llll}
\hline \multicolumn{3}{c}{ IEEE 14 bus system } & \\
\hline Algorithms & Congestion cost & Compensation cost & Final Cost \\
PSO & 5.5485 & 1508.4 & 14.812 \\
FF & 1.2185 & 1118.1 & 50.577 \\
GWO & 5.5485 & 1508.2 & 14.811 \\
ABC & 14.274 & 158.5 & 52.851 \\
WOA & 5.5174 & 1210.8 & 18.454 \\
Proposed method & 5.5485 & 1508.2 & 14.811 \\
& & IEEE 30 bus system & \\
PSO & 22 & 278.5 & 28.785 \\
FF & 22 & 275.15 & 28.755 \\
GWO & 22 & 588.1 & 25.881 \\
ABC & 22 & 588.1 & 25.881 \\
WOA & 22 & 282.55 & 28.825 \\
Proposed method & 22 & 585.87 & 25.851 \\
\hline
\end{tabular}

Table 2: Computational time of proposed and existing techniques

\begin{tabular}{lll}
\hline Algorithm & IEEE 14 bus system & IEEE 30 bus system \\
\hline PSO & 4.5818 & 22.145 \\
FF & 4.5828 & 28.49 \\
GWO & 8.4494 & 42.141 \\
ABC & 8.4458 & 48.149 \\
WOA & 1.141554 & 15.259 \\
Proposed method & 4.8482 & 15.414 \\
\hline
\end{tabular}


Rescheduling Based Congestion Management Method Using Hybrid Grey Wolf Optimization - Grasshopper Optimization Algorithm in Power System

\section{Conclusion}

This work was proposed a new Congestion Management technique (rescheduling basis) which has integrated a novel hybrid method called GWO-GOA to optimize the generating power of augmented generators in the bus system. The proposed GWO-GOA method was the hybridization idea of two methods called GWO and GOA that has aspired in ideal and exact Congestion Management yet with minimized cost of rescheduling. The proposed technique performance was evaluated with the existing techniques based rescheduling scheme regarding the analysis of cost. From the outcomes, it was analyzed that the proposed GWO-GOA technique was minimized all the revealed costs.

\section{Compliance with Ethical Standards}

Conflicts of interest: Authors declared that they have no conflict of interest.

Human participants: The conducted research follows the ethical standards and the authors ensured that they have not conducted any studies with human participants or animals.

\section{References}

[1] Fariborz Zaeim-Kohan, Hadi Razmi, Hasan Doagou-Mojarrad,"Multi-objective transmission congestion management considering demand response programs and generation rescheduling", Applied Soft Computing, Volume 70, September 2018, Pages 169-181.

[2] Aishvarya Narain, S. K. Srivastava, S. N. Singh,"Congestion management approaches in restructured power system: Key problems and challenges", The Electricity Journal, Volume 33, Problem 3, April 2020.

[3] Sadhan Gope, Arup Kumar Goswami, Prashant Kumar Tiwari, Subhasish Deb," Rescheduling of real power for congestion management with integration of pumped storage hydro unit using firefly algorithm", International Journal of Electrical Power \& Energy Systems, Volume 83, December 2016,Pages 434-442.

[4] S. S. Reddy, "Multi-Objective Based Congestion Management Using Generation Rescheduling and Load Shedding," in IEEE Transactions on Power Systems, vol. 32, no. 2, pp. 852-863, March 2017.

[5] S. Verma and V. Mukherjee, "Optimal real power rescheduling of generators for congestion management using a novel ant lion optimiser," in IET Generation, Transmission \& Distribution, volume. 10, number. 10, page no. 2548-2561, 772016.

[6] M. Mahmoudian Esfahani and G. R. Yousefi, "Real Time Congestion Management in Power Systems Considering Quasi-Dynamic Thermal Rating and Congestion Clearing Time," in IEEE Transactions on Industrial Informatics, volume. 12, number. 2, page no. 745-754, April 2016.

[7] Yesuratnam G, Thukaram D. Congestion management in open access based on relative electrical distances using voltage stability criteria. Electr Power Syst Res 2007;77:1608-18.

[8] Kumar A, Srivastava SC, Singh SN. Congestion management in competitive power market: a bibliographical survey. Electr Power Syst Res 2005;76:153-64.

[9] Yu Chien-Ning, Ilic MD. Congestion clusters-based markets for transmission management. IEEE Power Eng Soc 1999;2:821-32.

[10] Kumar A, Srivastava SC, Singh SN. A zonal congestion management approach using real and reactive power rescheduling. IEEE Trans Power Syst 2004;19:554-62.

[11] Deb Subhasish, Gope Sadhan, Goswami Arup Kumar. Congestion management considering wind energy sources using evolutionary algorithm. Electric Power Comp Syst 2015;43:723-32. 
[12] Naresh Kumar Yadav, " Rescheduling-based congestion management scheme using particle swarm optimization with distributed acceleration constants", Soft Computing, pp. 1-11, 2017.

[13] S. Mirjalili, S. M. Mirjalili, and A. Lewis, "Grey wolf optimizer," Advances in engineering software, volume. 69, page no. 46-61, 2014.

[14] S. Saremi, S. Mirjalili, and A. Lewis, "Grasshopper optimisation algorithm: theory and application," Advances in Engineering Software, volume. 105, page no. 30-47, 2017.

[15] W.Brajula and M.Bibin Prasad,"ODFF Opposition and Dimension based Firefly for Optimal Reactive Power Dispatch",Journal of Computational Mechanics, Power System and Control (JCMPS), Volume 1, Issue 1, October 2018.

[16] Vinolin V and S Vinusha,"Enhancement in Biodiesel Blend with the Aid of Neural Network and SAPSO",Journal of Computational Mechanics, Power System and Control (JCMPS), Volume 1, Issue 1, October 2018. 